\title{
Image Enhancement Techniques for Images at Blur Motion and Different Noises
}

\author{
Sahlah Abed Ali \\ sahlah80@gmail.com \\ Department of Computer Science \\ College of Computer Science and Mathematics \\ University of Mosul, Mosul, Iraq
}

Received on: $24 / 10 / 2018$

Accepted on: 24/01/2019

\begin{abstract}
Image restoration is an art to improve image quality by disposing of reducing the amount of noise and blurring present in the image. So it is one of the research titles of interest to the researchers. The images get degraded due to environmental conditions and atmospheric difference, it is therefore important to retrieve original images using different algorithms of image processing. There are widespread applications to restore images in our world today. In this research we present a new proposed method to manipulate image using median filter and has also applied inverse filter to image restoration, then combining the proposed method with inverse filter. The proposed method has been evaluated and compared with inverse Filter and with the merger by using different performance with parameters to verify efficiency and performances of these methods.

The experimental results on the test image will explain the capabilities of the proposed method to restored image, were the value of the image quality and PSNR (peak Signal to Noise ratio) is better compared with inverse filter, where values of PSNR for Lena Image is 70.3536 and for Girl Image is 71.5410 when using the proposed method of median filter, while values of PSNR when using inverse filter are 62.3225 and 62.6008 respectively when speckle noise.
\end{abstract}

Keywords: Noises, Blur, Image Restoration, Median Filter and Inverse Filter.

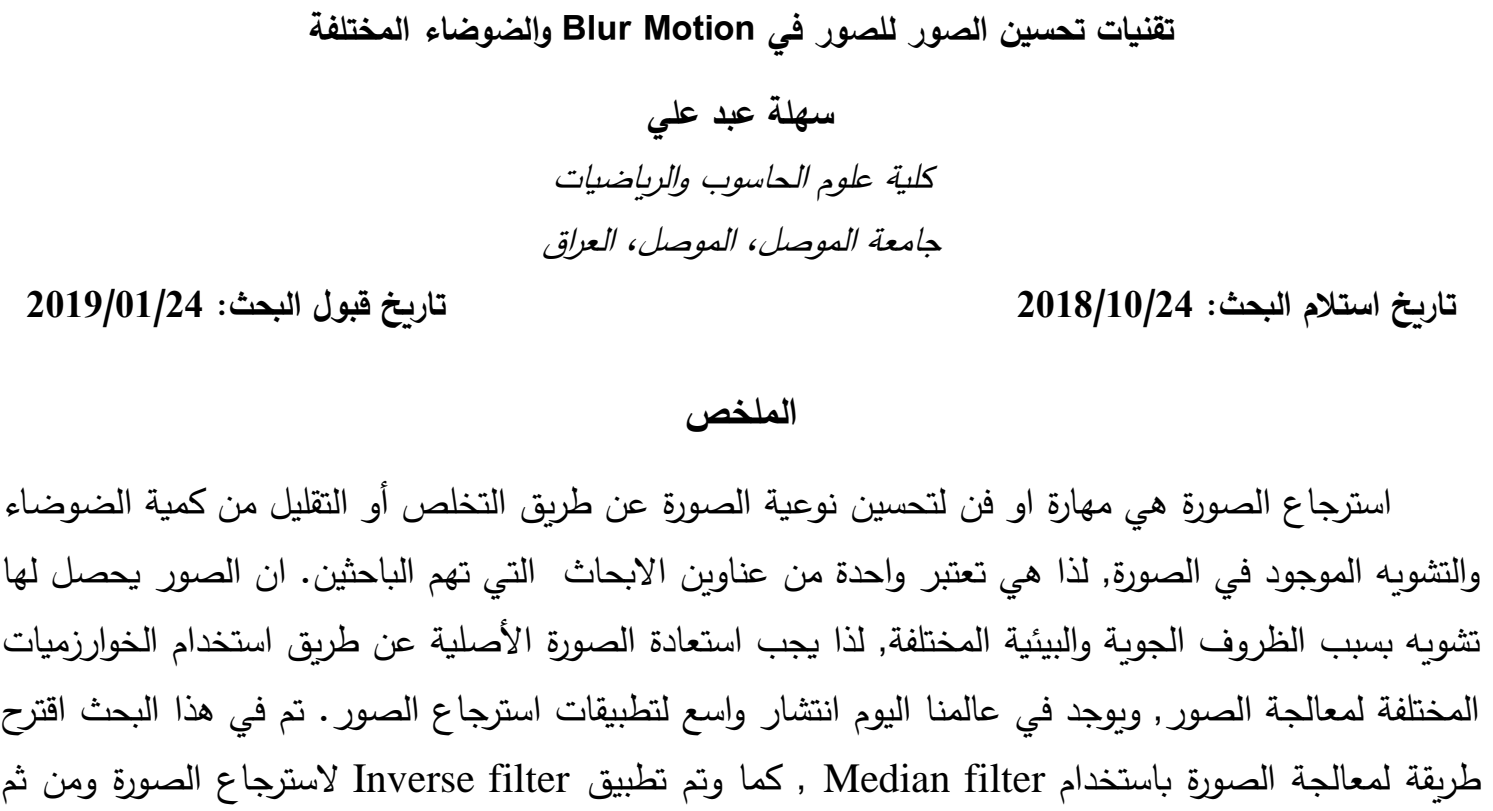


دمج الطريقة المتترحة مع Inverse filter. الطريقة المتترحة تم تقييمها ومقارنتها مع Inverse filter وطريقة

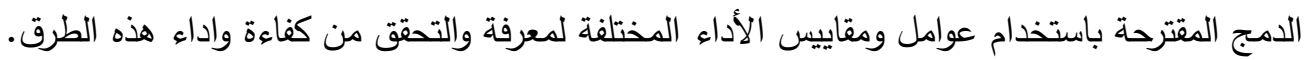

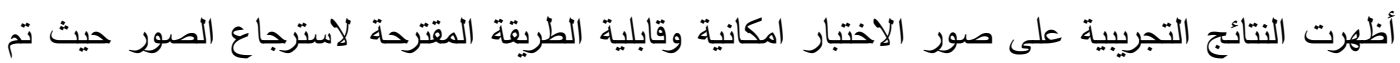

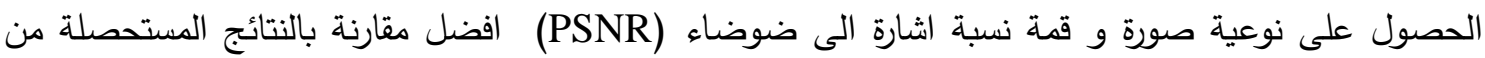

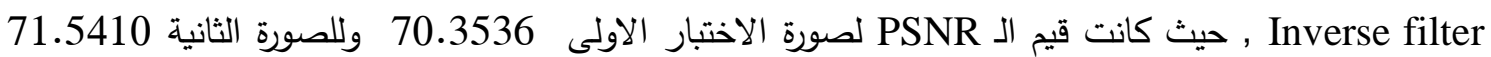

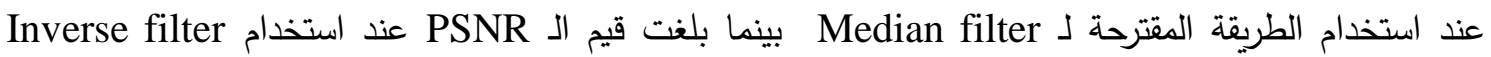

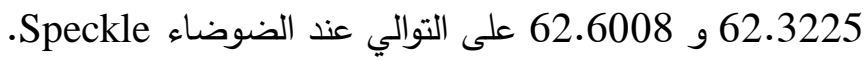

الكلمات المفتاحية: الضوضاء، التوهين، استرجاع الصورة، المرشح المتوسط، المرشح العكسي.

\section{Introduction}

Algorithms of image processing are essentially developed to overcome various problems, some of these include image restoration, image enhancement, image representation, image reconstruction, image preprocessing, image analysis and image data compression[1]. You can capture an image by using various digital devices and these may be corrupted due to several reasons, to handle these image restoration techniques must be used to recover original image. Image restoration is a process of removing or decreasing the degradation that occur on the image, and it represent field of engineering that manage with the approaches used to reconstruct the image with minimum degradation. During image capturing process, the image maybe corrupted due to camera misfocus or motion blurs if there is a proportional motion between camera and the captured image, so that recovering the image is essential for many technological applications [2]. There are several causes that make degradations but the more common type of it are Noise and Blurring. Noise is any undesirable information that infects the image while blurring is caused by the relative movement between the camera and the original scene. Image restoration techniques are interested in cancellation or decreasing a blurred and noisy from the corrupted image [3] . In this research a new method was proposed for median filter in order to find the median value, in addition, the inverse filter was applied and results obtained from it. The final filter was then combined with the proposed method and obtained the results of the merger, after that, all the results were compared and simulated by using some standard performance, standards such as Entropy, Root Mean Square Error (RMSE), Mean Absolute Error (MAE), Mean Square Error (MSE) and Peak signal to noise ratio (PSNR).

The paper is organized as: section 2 represents a brief review of the image restoration, section 3 description of the proposed work, section 4 gives Experimental results and Discussion finally section 5 explain the research conclusions.

\section{Methods And Techniques Of Image Restoration}

Image Restoration is the operation of recovering the original image from the degraded image by taking a corrupted/noisy image and eliminate and correct errors in order to retrieve the original images . There are many different applications that use the image restoration like Scientific areas, Medical applications, Remote Sensing, Astronomy, Military, Restoration of Compressed Images, Film industry etc. Restoration of Images deals with improved appearance of an image [3][4]. The degradation model consists of two parts, the degradation function and the noise function, whereas $I(i, j)$ is The original input and it is a two-dimensional image. $\mathrm{H}$ represents the system where the images are operated on, the output of it is addition with 
the noise $\eta(\mathrm{i}, \mathrm{j})$ and after the addition you can get $\mathrm{g}(\mathrm{i}, \mathrm{j})$ which represent the degraded image. Applying Restoration filters on the given $g(i, j)$ and $\mathrm{H}$ get the restored image $I^{\wedge}(i, j)$. The model of degradation can be expressed mathematically as [1].

$$
g(i, j)=h(i, j) * I(i, j)+\eta(i, j)
$$

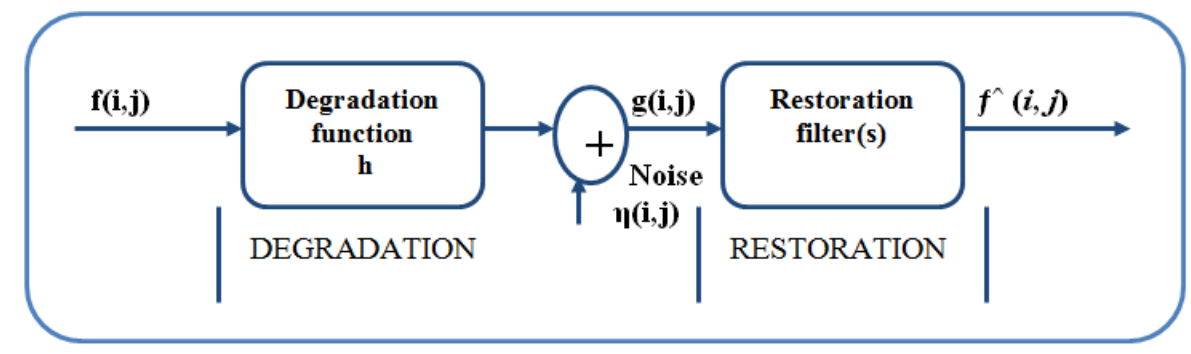

Fig.1. Model of Image Degradation and Restoration

Canceling the noise that occurred in the image is one of the main tasks done in image processing and computer vision. There are various restoration techniques that are used to cancel or reduce noises from degraded image and reconstruct the original image [5]. Proposed method in this paper was introduced by adopting method Median Filtering in addition to use Inverse filter and merge this filter with a novel method.

\section{Proposed Work}

Digital images are discomposed by various kinds of noise detections and removal of this noises plays a crucial act in restoration techniques. Image restoration is usually the first step of the whole process of the image processing. The motion blur with noises that is used in this paper are the following[6]:

The speckle noises: Are granular noises that are already present in the quality of the synthetic aperture radar (SAR) and active radar images.

The gaussian noises: It is statistical noise with PDF (a probability density function) equal to normal distribution, Gaussian refers to the way gray values are distributed .

Salt and Pepper noises: Are data leakage noise, usually referred to as impulse noise. Image that contains Salt \& pepper noises will contain dark pixels in bright areas and bright pixels in dark areas. Noises are caused by adapter errors and transmission bit errors .

The Motion Blur: is the proportional movement between the scene and the photographic device such as sudden change in the scene or device make the image look like moving by adding blur in a particular direction [7].

\section{Image Restoration Using Inverse Filter}

Inverse filtering is a deterministic and direct method for image restoration, it is the easiest and fastest way to restore the corrupt image if can build a good pattern of the blurring that corrupt an image. But unfortunately this filter is high pass filter where it responds very badly to any noise present in the image [8][9]. The inverse filtering method used in this paper is a thresholding method. A blurred image model can be define in spatial domain by:

$$
g(i, j)=I(i, j) * h(i, j)
$$

where $\mathrm{f}$ is the original image, $* *$ is $2-\mathrm{D}$ convolution and $\mathrm{h}$ is PSF (point spread function) while a blurred image is $\mathrm{g}$. In order to restore the original image, you just need to convolved a blurred image with a high pass filter [8][9].

$$
I(i, j)=h(i, j) * g(i, j)
$$

In this work take Fast Fourier Transform of $\mathrm{h}$ as follow:

$$
\mathrm{hf}=\mathrm{FFT} 2(\mathrm{~h})
$$


to get a high pass filter we must only reverse all the elements of hf, this is the ideal case, but we will notice here a lot of the elements in hf have values zero or very close to it and some of other elements give us either very high values or some infinite values. In this case we will need to set some sort of a threshold to avoid these values by[8][9]:

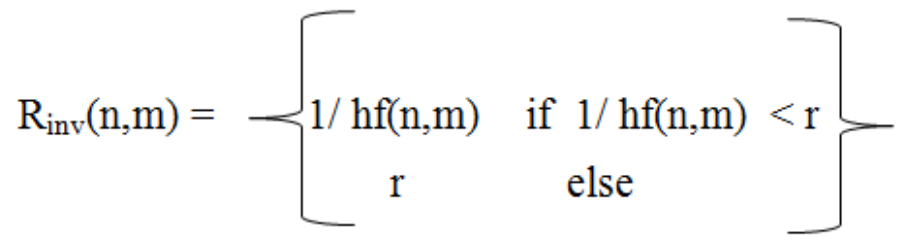

So, $r$ is the higher while $R_{\text {inv }}$ is the closer to the inverse filter. This is called regularized inverse filter that implemented in our work.

\section{Algorithm of Inverse Filter:-}

Step1- Read a color image into the MATLAB environment.

Step2- Using the function (PSF = fspecial('Motion', Len, Theta)) to create the PSF

Step 3- Convolution the image with PSF to create a simulated blur in it (blurry image), which can be implemented with FFT2.

Step 4- Create the noise and add it with a blurry image.

Step 5- Use Inverse filter to restore the blurred noisy image by dividing it by the FFT2 of $h$ (PSF) and performing an IFFT2 of the result to reconstruct image.

\section{Image Restoration Using A proposed Median Filter}

It is a temporal and spatio-temporal filters work good to reduce added noise. Median filters are widely used to reduce impulsive or "salt and pepper" and speckle noise. It is a non-linear filter and fit in the generic category of order statistic filters which operate on a window of pixels. In the median filter we replace the pixel value with the median of surrounding neighbouring to it, however, all pixel values in the window must first be sorted in ascending order, and the pixel value should be replaced by middle pixel value. Median filter is common because of advantages inherent to find the middle value where it keeps edges well and reduce distortion in the image than linear filters. However, some of fine details can be lost depending on the window size of the filter, this is due to the nature of the median operation. So implementations use a small window size of $3 \times 3$ is preferred [10][11].

We proposed a new method in this paper in order to find the median value and is a clockwise route method. The basic idea from the propose method behind filter is an application median technique several time for any pixels of the image. Clockwise route method maintain the edges and gives better result than basic version of the median filter because there are fifth step operation to find the median value: values from different directions are ranked separately, as in the example for window size 5x5: 


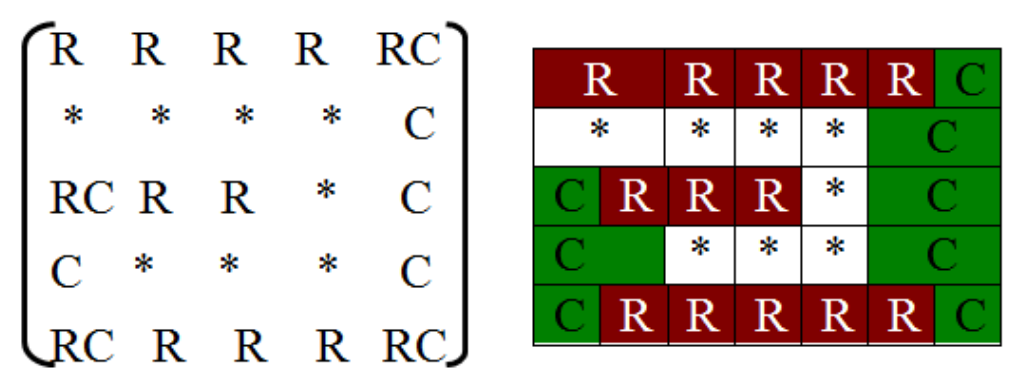

Fig. 2. The Median Value for the Proposed Method.

\section{Work steps of the proposed Clockwise route method:}

Step 1- Follow the route and pick up the first row elements;

Step 2- Order elements and Take the middle element;

Step 3- Follow the route and pick up the last column elements;

Step 4- Order elements and Take the middle element;

Step 5- Follow the route and pick up the last row elements;

Step 6- Order elements and Take the middle element;

Step 7- Pick up result in point 2, 4 and 6;

Step 8- Order elements and Take the middle element;

Step 9- Follow the route and pick up half of the first column elements;

Step 10- Order elements and Take the middle element;

Step 11- Follow the route and pick up half of the middle row elements;

Step 12- Order elements and Take the middle element;

Step 13- Pick up result in point 8, 10 and 12;

Step 14- Order elements and Take the middle element;

Initially window is put over an element at the edge but some part of the window will be blank. To fill the void, the image must be stretched by adding the rows at the top and bottom of the image and also adding columns to the right and left of it. By this way we have preserved the corners. In this paper I apply windows $3 \times 3$ and $5 \times 5$ median filters, the advantage of the proposed method is better than the standard median filter.

\section{Discussion Of The Experimental Results}

In this paper we are use MATLAB7.11(R2010b) version to simulate the results. The Matlab functions allow us to add different classical noises to an image, So used this function which is shown in the figures below, the test images that were used in this paper are Lena.bmp of size 256x 256 and Girl.bmp of size 196x262. In order to evaluate the performance of the proposed method we applied it on test image and obtained the results and then applied the inverse filter on the test image also and also obtained the results. The results of the inverse filter were then merged with the proposed method of the median filter by applying the proposed method to the image resulting from the inverse filter and obtaining the results also, all results obtained were then compared, all this work in order to improve images and get the best results desired. the results of which are shown in the figures from figure (2) to figure (10) and tables $(1,2,3)$ are 
below. Several criteria in terms of MSE (mean square error), PSNR (peak signal-tonoise ratio) [12], MAE (mean absolute error), RMSE (Root Mean Square Error) and Entropy [3] are presented to assess the quantitative performance of our work, which are shown in tables $(1,2,3)$ and they are given as:

PSNR $=10 \log _{10}\left[\left(\mathrm{~d}(\mathrm{x}, \mathrm{y})_{\max }-\mathrm{d}(\mathrm{x}, \mathrm{y})_{\min }\right)^{2} / \mathrm{MSE}\right]$

Where, MSE symbolizes the Error between the original and the restored images, and is given as:

$$
\mathrm{MSE}=1 / \mathrm{MN} \sum_{i=1}^{M} \sum_{j=1}^{N}\left(f(\mathrm{i}, \mathrm{j})-f^{\wedge}(\mathrm{i}, \mathrm{j})\right)^{2}
$$

Where $\mathrm{M}$ and $\mathrm{N}$ are the width and height of Image, $f^{\wedge}$ Restored Image and $f$ Original Image. While RMSE is the square of MSE.

MAE $=\operatorname{sum}(\operatorname{abs}(f(:)-$ blurred image $(:))) / \operatorname{numel}(f)$

While Entropy is used to measure the information content of an image. It is sensitive to noise and other unwanted fluctuations. An image with high information content will have high entropy.

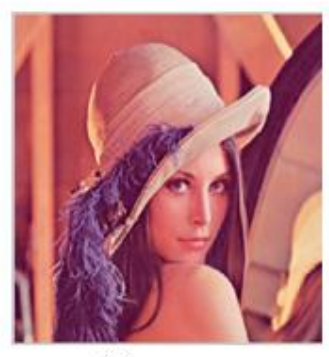

(a)

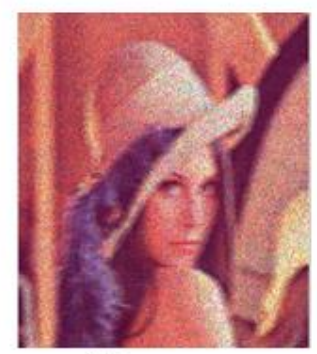

(b)

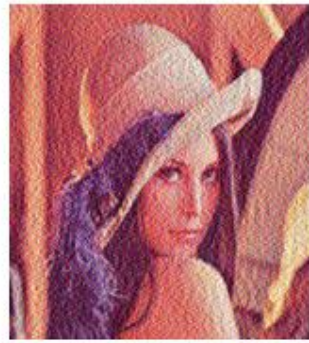

(c)

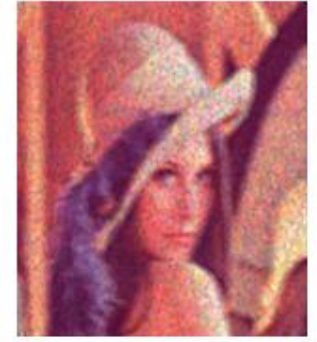

Restored Image By proposed median filter

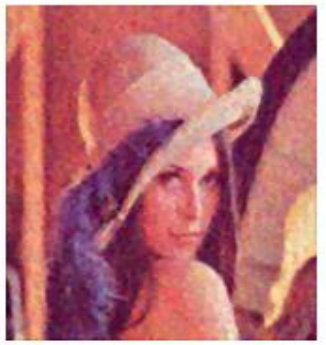

Restored Image By merge proposed median with inverse filter]

Fig.(2): (a) Original Image, (b) Image with Noise Speckle \& Blur Motion and (c) Reconstructed image of Lena. 


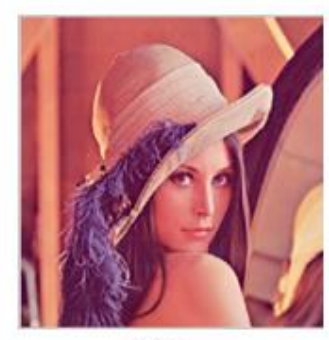

(a)

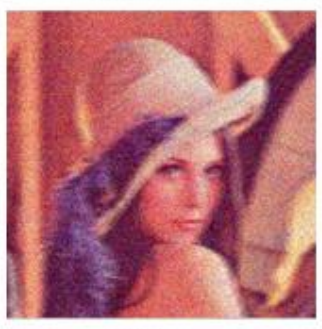

(b)

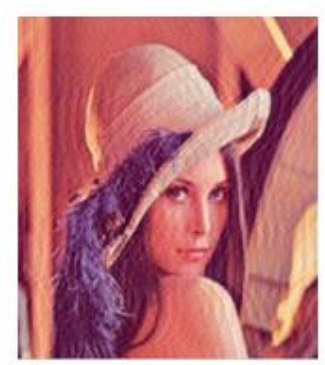

(c) Inverse Filter

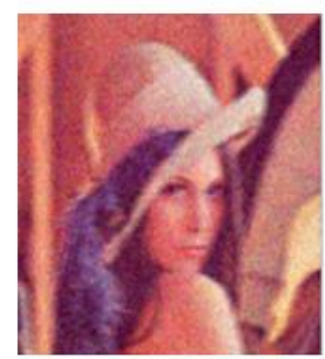

Restored Image By proposed median filter

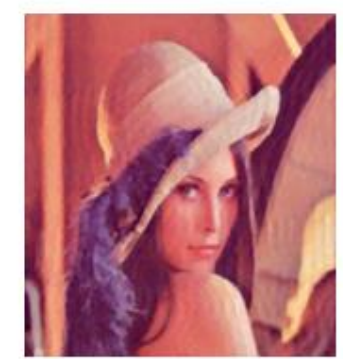

Restored Image By merge proposed median with inverse filter]

Fig.(3): (a) Original Image, (b) Image with Noise Gaussian \& Blur Motion and (c) Reconstructed image of Lena.

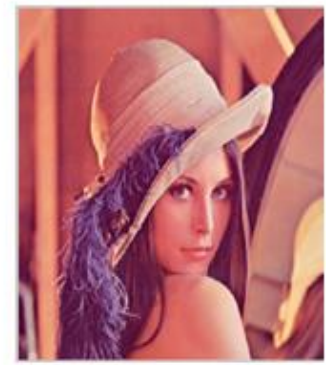

(a)

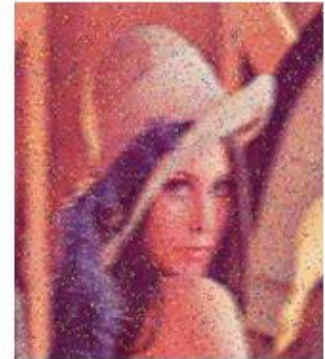

(b)

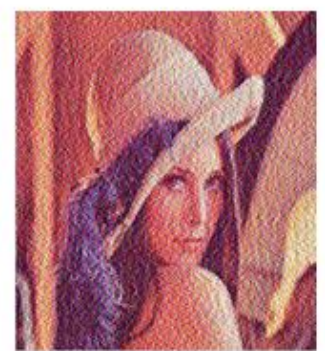

(c) [ Restored Image By Inverse Filter

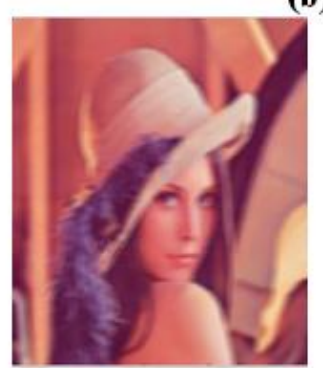

Restored Image By proposed median filter

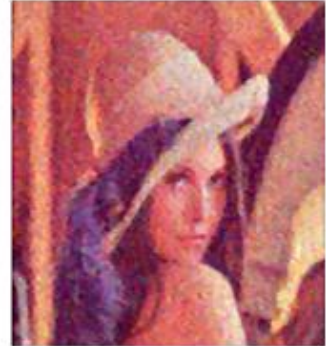

Restored Image By merge proposed median with inverse filter]

Fig.(4): (a) Original Image, (b) Image with Noise "Salt \& pepper" and Blur Motion and

(c) Reconstructed image of Lena. 


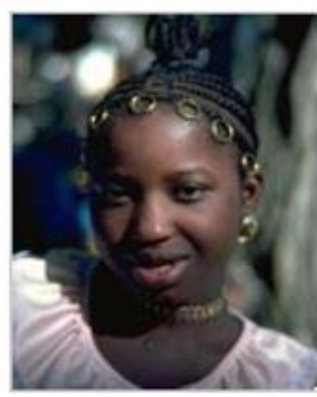

(a)

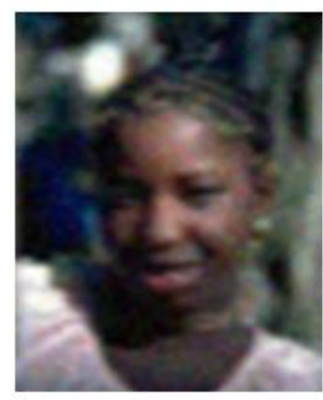

(b)

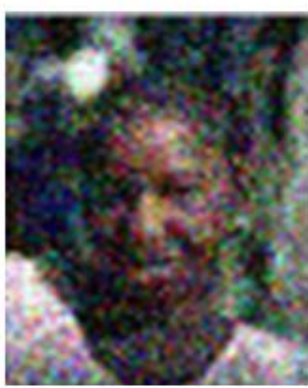

(c) [ Restored Image By Inverse Filter

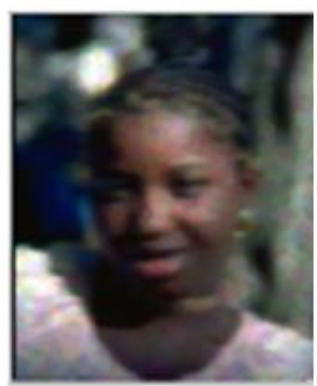

Restored Image By proposed median filter

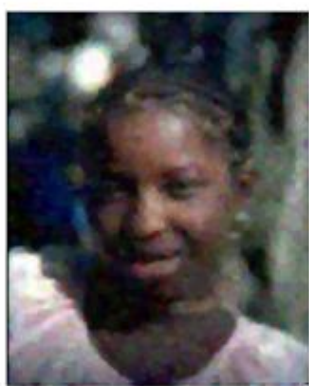

Restored Image By merge proposed median with inverse filter]

Fig.(5): (a) Original Image, (b) Image with Speckle \& Blur Motion and (c)

Reconstructed image of Girl.

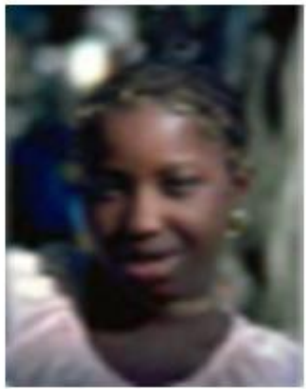

(a)

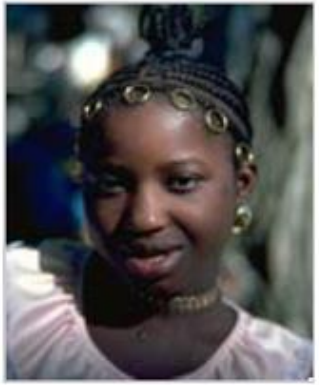

(b)

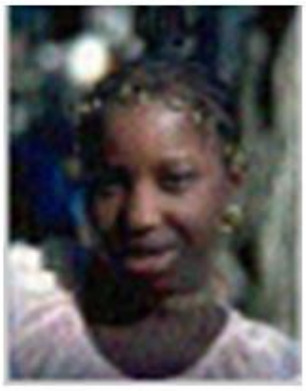

(c) [ Restored Image By Inverse Filter

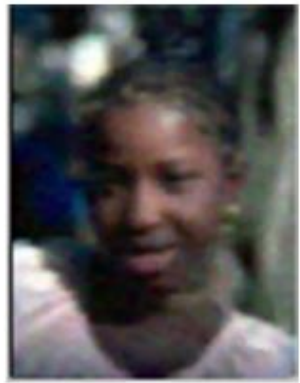

Restored Image By proposed median filter

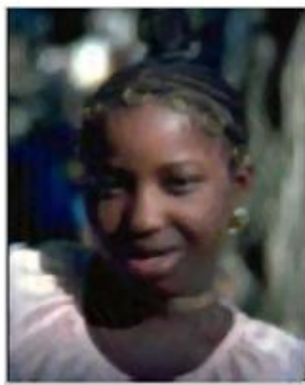

Restored Image By merge proposed median with inverse filter]

Fig.(6): (a) Original Image, (b) Image with Gaussian \& Blur Motion and (c)

Reconstructed image of Girl. 

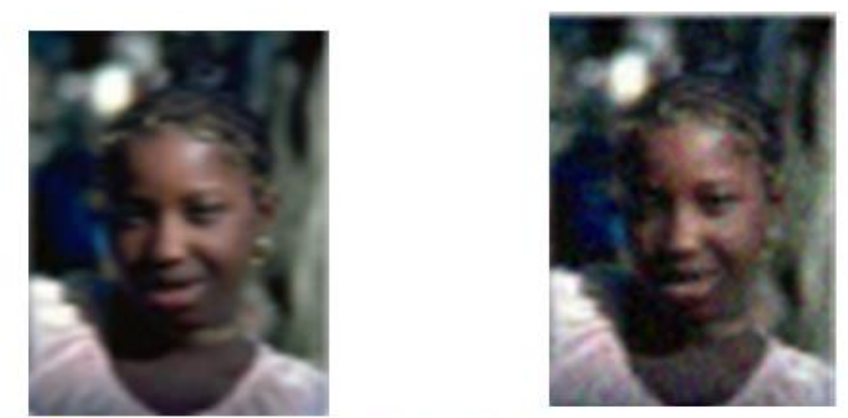

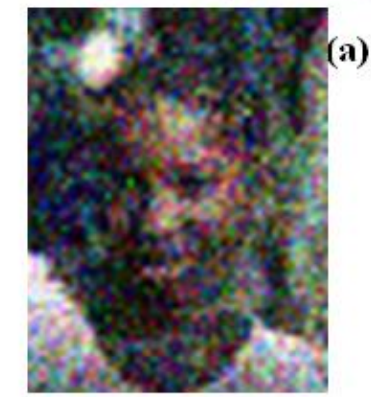

(c) [ Restored Image By Inverse Filter

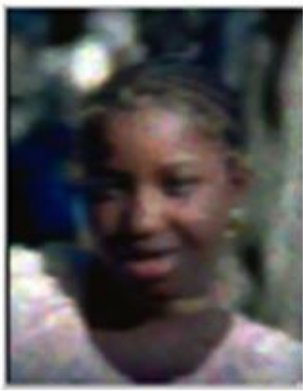

Restored Image By proposed median filter

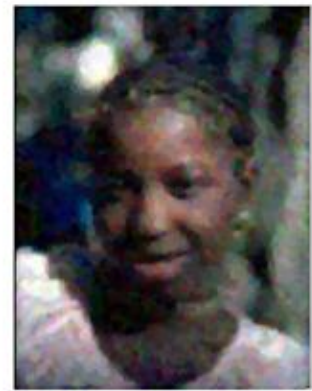

Restored Image By merge proposed median with inverse filter]

Fig.(7): (a) Original Image, (b) Image with "Salt \& pepper" and Blur Motion and (c) Reconstructed image of Girl.

TABLE1. Evaluation the performance criteria to assess images restoration with Speckle noise for Lena and Girl images.

\begin{tabular}{|c|c|c|c|c|}
\hline $\begin{array}{r}\text { Test } \\
\text { images }\end{array}$ & Criteria $\backslash$ Filters & $\begin{array}{r}\text { Inverse } \\
\text { Filter }\end{array}$ & $\begin{array}{r}\text { A proposed Median } \\
\text { Filter }\end{array}$ & $\begin{array}{r}\text { Merge a proposed } \\
\text { Median with Inverse } \\
\text { Filter }\end{array}$ \\
\hline \multirow{5}{*}{ 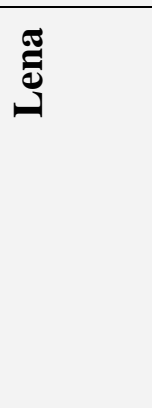 } & Entropy & 7.7086 & 7.6809 & 7.7791 \\
\hline & $R M S E$ & 0.1952 & 0.0774 & 0.0728 \\
\hline & $M A E$ & 0.1548 & 0.0938 & 0.0760 \\
\hline & PSNR & 62.3225 & 70.3536 & 70.8933 \\
\hline & $M S E$ & 0.0381 & 0.0060 & 0.0053 \\
\hline \multirow{3}{*}{ 氙 } & Entropy & 7.2253 & 7.5101 & 7.5554 \\
\hline & $R M S E$ & 0.1890 & 0.0675 & 0.0698 \\
\hline & MAE & 0.0725 & 0.0587 & 0.0725 \\
\hline
\end{tabular}




\begin{tabular}{|l|r|r|r|r|}
\hline \multirow{2}{*}{ PSNR } & 62.6008 & 71.5410 & 71.2511 \\
\cline { 2 - 5 } & MSE & 0.0357 & 0.0046 & 0.0049 \\
\hline
\end{tabular}

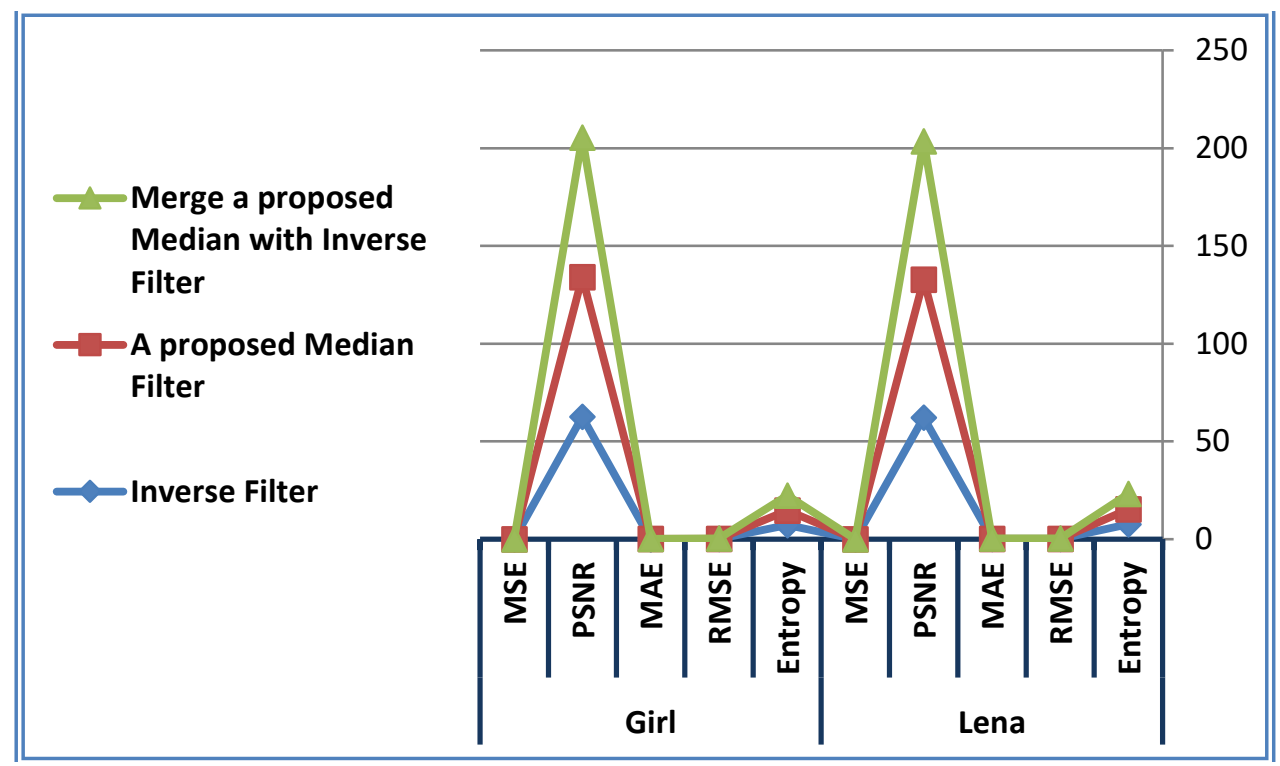

Fig.(8): The Comparison of the images restoration with Speckle noise for Lena and Girl images.

TABLE2. Evaluation the performance criteria to assess images restoration with Gaussian noise for Lena and Girl images.

\begin{tabular}{|c|c|c|c|c|}
\hline $\begin{array}{r}\text { Test } \\
\text { images }\end{array}$ & Criteria \Filters & $\begin{array}{r}\text { Inverse } \\
\text { Filter }\end{array}$ & $\begin{array}{r}\text { A proposed Median } \\
\text { Filter }\end{array}$ & $\begin{array}{r}\text { Merge a proposed } \\
\text { Median with Inverse } \\
\text { Filter }\end{array}$ \\
\hline \multirow{5}{*}{ 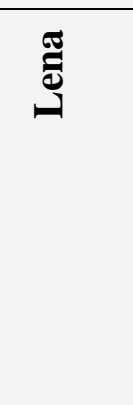 } & Entropy & 7.8004 & 7.6953 & 7.7215 \\
\hline & RMSE & 0.0567 & 0.0701 & 0.0385 \\
\hline & $M A E$ & 0.0449 & 0.0890 & 0.0653 \\
\hline & PSNR & 73.0541 & 71.2124 & 76.4307 \\
\hline & $M S E$ & 0.0032 & 0.0049 & 0.0015 \\
\hline \multirow{2}{*}{$\overline{1}$} & Entropy & 7.5651 & 7.5564 & 7.5531 \\
\hline & RMSE & 0.0518 & 0.0680 & 0.0590 \\
\hline
\end{tabular}




\begin{tabular}{|r|r|r|r|r||}
\hline & MAE & 0.0632 & 0.0801 & $\mathbf{0 . 0 6 3 2}$ \\
\cline { 2 - 5 } & $\boldsymbol{P S N R}$ & 73.8371 & 71.4869 & $\mathbf{7 5 . 7 2 0 1}$ \\
\cline { 2 - 5 } & & 0.0027 & 0.0046 & $\mathbf{0 . 0 0 3 5}$ \\
\hline
\end{tabular}

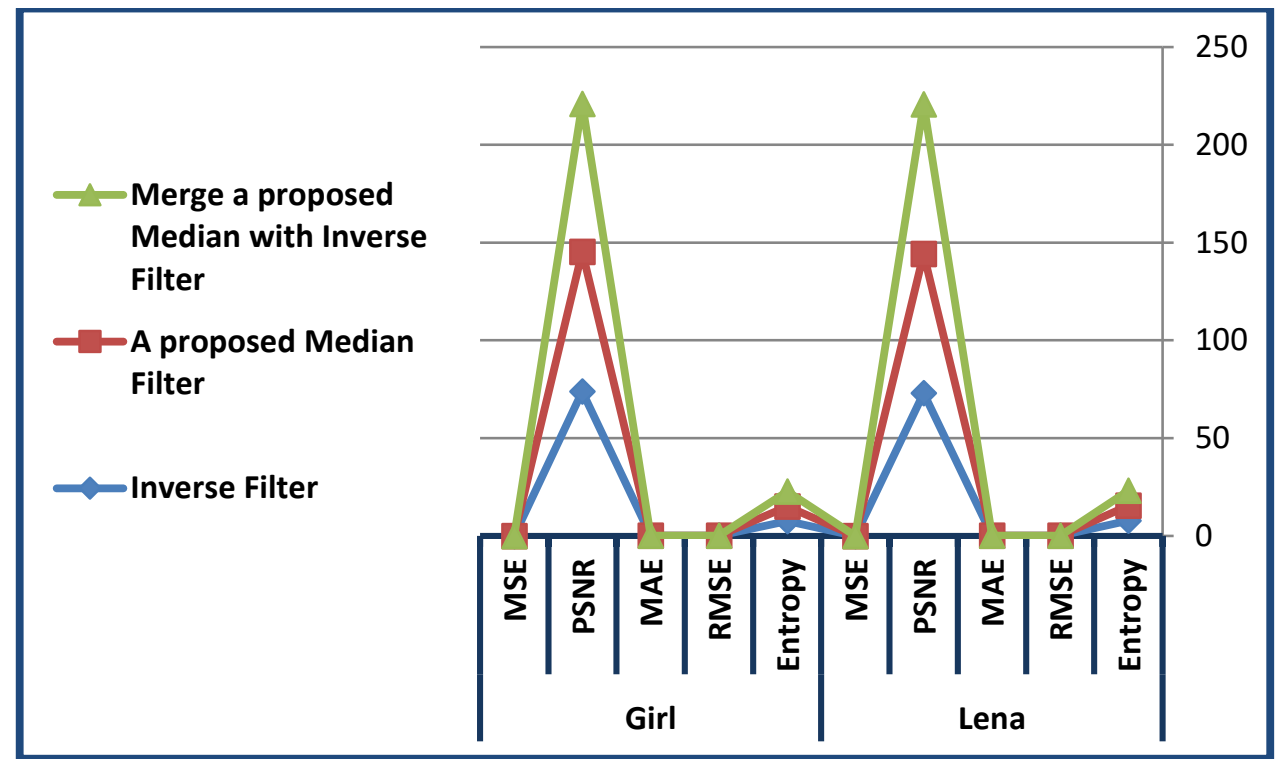

Fig.(9): The Comparison of the images restoration with Gaussian noise for Lena and Girl images.

TABLE 3. Evaluation the performance criteria to assess image restoration with salt \& pepper noise for Lena and Girl images.

\begin{tabular}{|c|c|c|c|c|}
\hline $\begin{array}{r}\text { Test } \\
\text { images }\end{array}$ & Criteria $\backslash$ Filters & $\begin{array}{r}\text { Inverse } \\
\text { Filter }\end{array}$ & $\begin{array}{r}\text { A proposed Median } \\
\text { Filter }\end{array}$ & $\begin{array}{r}\text { Merge a proposed } \\
\text { Median with Inverse } \\
\text { Filter }\end{array}$ \\
\hline \multirow{5}{*}{ હ્ّ } & Entropy & 7.5691 & 7.6270 & 7.7887 \\
\hline & RMSE & 0.2419 & 0.0561 & 0.0852 \\
\hline & $M A E$ & 0.1930 & 0.0560 & 0.0808 \\
\hline & PSNR & 60.4599 & 73.1557 & 69.5233 \\
\hline & $M S E$ & 0.0585 & 0.0031 & 0.0073 \\
\hline \multirow{4}{*}{$\bar{B}$} & Entropy & 7.0880 & 7.5318 & 7.5545 \\
\hline & $R M S E$ & 0.2347 & 0.0603 & 0.0743 \\
\hline & $M A E$ & 0.0773 & 0.0464 & 0.0772 \\
\hline & PSNR & 60.7222 & 72.5243 & 70.7149 \\
\hline
\end{tabular}




\begin{tabular}{|l|r|r|r|r|}
\hline & MSE & 0.0551 & $\mathbf{0 . 0 0 3 6}$ & 0.0055 \\
\hline
\end{tabular}

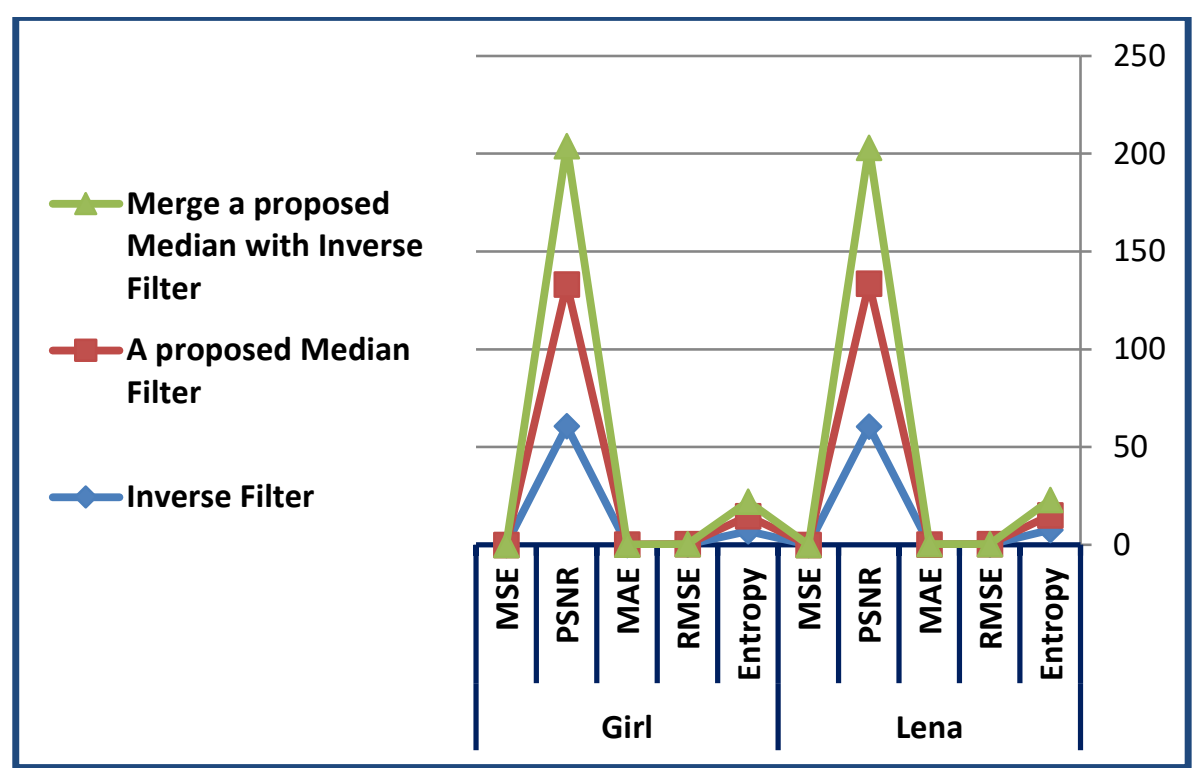

Fig.(10): The Comparison of the images restoration with salt \& pepper noise for Lena and Girl images.

\section{Conclusion}

This paper focuses on restoring the original image with minimum degradation in order to produce the image with high-quality by canceling or reducing the noises and Blurs from the degraded image. From the results we show that the performance of the proposed Median filter method is very good while the performance of Inverse filter is acceptable. In order to improve the results obtained and to obtain the best results the proposed Median filter method was merged with the Inverse filter, where the proposed method was applied to the image retrieved (resulting) from inverse filter. From the experimental results in the tables, show that the performance of the proposed method after merge is very good in case of the speckle and Gaussian noises, while the performance of the proposed method is very good in case of 'salt \& pepper' noises and also speckle noise compare with invers filter. 


\section{REFERENCES}

[1] R. C. Gonzalez and R. Woods, "Digital Image Processing", Addison-Wesley Publishing Company, 1993.

[2] S. K. Satpathy, S. Panda, K. K. Nagwanshi and C. Ardil, "Image Restoration in Non-Linear Filtering Domain using MDB approach", World Academy of Science, Engineering and Technology International Journal of Computer and Information Engineering Vol:7, No:2, 2013.

[3] Deepa Puri , Prof. Santosh Kumar Mishra, "Analysis of Image Restoration Techniques at Different Noises", Computer Science and Engineering, VNSIT Bhopal, International Journal of Computer Science and Information Technologies (IJCSIT), Vol. 8 (3) , 2017, 439-442.

[4] CharuKhare, Kapil Kumar Nagwanshi, "Image Restoration Technique with Non Linear Filter", International Journal of Advanced Science and Technology Vol. 39, February, 2012.

[5] Jyh-Yeong Chang and Jia-Lin Chen, " Classifier-Augmented Median Filters for Image Restoration", IEEE TRANSACTIONS ON INSTRUMENTATION AND MEASUREMENT, VOL. 53, NO. 2, APRIL 2004.

[6] Geoff Dougherty (2010) "Digital Image Processing for Medical Applications," second ed., Cambridge university press.

[7] CharuKhare \& Kapil Kumar Nagwanshi, "Implementation And Analysis Of Image Restoration Techniques", International Journal of Computer Trends and Technology- May to June Issue 2011.

[8] http://www.owlnet.rice.edu/ elec539/Projects99/BACH/proj2/inverse.html

[9] Mr. Firas Ali, " Image restoration using regularized inverse filtering and adaptive threshold wavelet denoising ", Mechatronics Engineering Department ,Al- Khwarizmi College of Engineering, University of Baghdad, Al- Khwarizmi Engineering Journal, Vol.3, No.1 , pp 48-62 (2007)

[10] Shawn R. Jackson, "Image sequence restoration by median filtering", Rochester Institute of Technology, Thesis of Master of Science in Computer Engineering, http://scholarworks.rit.edu/theses, 2004.

[11] Rakesh M.R, Ajeya B and Mohan A.R, " Hybrid Median Filter for Impulse Noise Removal of an Image in Image Restoration ", International Journal of Advanced Research in Electrical, Electronics and Instrumentation Engineering, Vol. 2, Issue 10, October 2013.

[12] D.Gnanadurai, and V.Sadasivam, "An Efficient Adaptive Thresholding Technique for Wavelet Based Image Denoising", International Journal of Signal Processing Volume 2 Number 2, 2005 ISSN 1304-4494. 